\title{
On the Relationship Between Coronal Magnetic Decay Index and CME Speed
}

\author{
Yan $\mathrm{Xu}^{1}$, Chang $\mathrm{Liu}^{1}, \mathrm{Ju}^{\mathrm{J}} \mathrm{Jing}^{1}$, and Haimin Wang ${ }^{1}$ \\ 1. Space Weather Research Lab, Center for Solar-Terrestial Research, \\ New Jersey Institute of Technology \\ 323 Martin Luther King Blvd, Newark, NJ 07102-1982 \\ yx2@njit.edu
}

August 14, 2018

\begin{abstract}
Numerical simulations suggest that kink and torus instabilities are two potential contributors to the initiation and prorogation of eruptive events. A magnetic parameter named decay index (i.e., the coronal magnetic gradient of the overlying fields above the eruptive flux ropes) could play an important role in controlling kinematics of eruptions. Previous studies have identified a threshold range of the decay index that distinguishes between eruptive and confined configurations. Here we advance the study by investigating if there is a clear correlation between the decay index and CME speed. 38 CMEs associated with filament eruptions and/or two-ribbon flares are selected using the $\mathrm{H} \alpha$ data from the Global $\mathrm{H} \alpha$ Network. The filaments and flare ribbons observed in $\mathrm{H} \alpha$ associated with the CMEs help to locate the magnetic polarity inversion line, along which the decay index is calculated based on the potential field extrapolation using MDI magnetograms as boundary conditions. The speeds of CMEs are obtained from the LASCO C2 CME catalog available online. We find that the mean decay index increases with CME speed for those CMEs with a speed below $1000 \mathrm{~km} / \mathrm{s}$, and stays flat around 2.2 for the CMEs with higher speeds. In addition, we present a case study of a partial filament eruption, in which the decay indexes show different values above the erupted/non-erupted part.
\end{abstract}

Subject headings: Sun: activity — Sun: coronal mass ejections (CMEs) — Sun: filaments, prominences — Sun: magnetic topology 


\section{Introduction}

A coronal mass ejection (CME) is a sudden large-scale eruption from the solar surface to the interplanetary space. The total mass carried by a CME could reach the order of $10^{13} \mathrm{~kg}$ (Chen et al. 2001) and the observed speed is as high as $3387 \mathrm{~km} / \mathrm{s}$ according to the online catalog by the Large Angle and Spectrometric Coronagraph (LASCO). The outward ejections contain not only massive plasmas but also magnetic fields. An earth-oriented CME can disrupt the Earth's magnetosphere or even as deep as the Earth's surface.

Statistical studies (Moon et al. 2002; Jing et al. 2004) have found that CMEs are often associated with other phenomena of solar activity, such as filament eruptions and solar flares. From a theoretical aspect, they are now believed to be different manifestations of a single eruptive event (e.g., Priest \& Forbes 2002), in which magnetic reconnection is a key process. In an equilibrium state, a filament may be supported by the magnetic flux rope (Antiochos et al. 1999; Fan \& Gibson 2007; Török \& Kliem 2005). However, the kink and torus instabilities, determined by the core field of magnetic flux rope and overlaying field (named strapping field), could lead filaments to erupt. The kink instability is measured by the number of magnetic twist. When the twist exceeds a certain threshold, for instance $2.5 \pi$ to $3.5 \pi$ (Hood \& Priest 1981; Fan \& Gibson 2003; Török et al. 2004), the filament would become unstable and eruptive. On the other hand, filament eruptions can also be triggered by torus instability, as a result of the broken of Shafranov equilibrium when the field strength decreases faster (Kliem \& Török 2006; Török \& Kliem 2005). In this respect, knowledge of gradient of the strapping field is crucial for understanding the physical mechanisms of solar eruptive events.

To quantitatively describe how fast the strapping field decays, a decay index is defined as: $n=-d \log \left(B_{t}\right) / d \log (h)$ (Kliem \& Török 2006), in which $B_{t}$ is the strength of the strapping field in transverse direction and $h$ is the radial height above the photosphere. According to this definition, the larger value of decay index indicates that the overlying field strength decreases faster. In practice, the field strengths at different heights can be obtained by extrapolating coronal field under the assumption of potential field. A typical range of decay index $n$ falls into $1.1 \sim 2.0$ (Bateman 1978; Démoulin \& Aulanier 2010; Fan \& Gibson 2007; Schrijver et al. 2008). Based on a sample of ten events, Liu (2008) find that "for a failed eruption, the field strength is about a factor of 3 stronger than that for a full eruption". Decay index is usually measured along the polarity inversion line (PIL). In statistical studies such as Liu (2008), all the decay indexes along a certain PIL were averaged to yield one single value representing the magnetic configuration of the corresponding active region. However, in some particular events, the decay index varies significantly along the PIL. Liu et al. (2010a) analyzed an eruptive event on 2005 Jan 15, in which a filament erupted partially. They 
found that the decay index above the region of failed filament eruption are much smaller than those above eruptive regions. More recently, Guo et al. (2010) analyzed another failed event on 2005 May 27 and further confirmed that the kink instability initiated this failed event, but confined by above strapping fields.

The main objective of this paper is to present the statistical study of correlation between decay index and CME speed. We collected 38 events and calculated the decay index of each event. A positive correlation between the CME speed and the decay index is found. In addition, we present analysis of a partial eruption on 2000 Sep 20 observed by Big Bear Solar Observatory (BBSO). Decay indexes along the PIL are derived using Michelson Doppler Imager (MDI) magnetograms and the different decay indexes above erupte/non-erupted region were found.

\section{Observations and Measurements}

In order to calculate the decay index, extrapolation of magnetic field are often used as coronal field measurement is not available. Non-linear force free field (NLFFF) extrapolation of high-quality vector magnetograms is believed to represent the $3 \mathrm{D}$ coronal magnetic field accurately. On the other hand, it is usually reasonable to assume that the strapping field may be more objectively described by potential fields, as fields turn potential above certain height even in complicated active regions (Jing et al. 2008). In addition, NLFFF method requires vector magnetograms with large field-of-view (FOV), which are unavailable prior to the launch of Helioseismic and Magnetic Imager on the Solar and Heliospheric Observatory (SDO/HMI). Therefore, the usage of potential field extrapolation that only requires the lineof-sight magnetograms, will expand the number of feasible events tremendously. Previous studies also suggest that the coronal field reconstructed under the NLFFF assumption may approach a potential configuration above an altitude of about $30 \mathrm{Mm}$ (e.g., Jing et al. 2008). In this study, potential field is computed using a Green's function method (Chiu \& Hilton 1977; Metcalf et al. 2008). The extrapolation was constructed by 100 grids in the vertical direction above the photosphere. The grid space is one pixel. With the MDI's 1.98" pixel resolution, we are able to construct a 3D magnetic field with a vertical dimension of about $140 \mathrm{Mm}$. In previous studies of Liu (2008) and Liu et al. (2010a), the strapping fields were believed to dominate in a range of $42 \sim 105 \mathrm{Mm}$. Xu et al., 2010 also found that a filament eruption mainly starts from 20 to $60 \mathrm{Mm}$ using the stereoscopy method with EUV and $\mathrm{H} \alpha$ data. Therefore, in this study, decay indexes are calculated in the same height range of 42 $\sim 105 \mathrm{Mm}$ as in the previous studies.

It is well known that the filaments or flux ropes reside above the PILs which divide 
opposite magnetic polarity fluxes (Liu 2008). Therefore, the decay indexes along the PIL in principle represent the majority of decay of strapping field and were commonly calculated in previous analysis (Liu 2008; Guo et al. 2010). Typically, a PIL is identified by choosing the points with zero Gauss but large gradient on the magnetograms. However, in most of events, the PIL extends far away from the center of an active region or filament of interest, leading to substantial uncertainties in the averaged decay index along the entire PIL. Moreover, there are some sections of PIL belong to "quiet" regions that do not contribute to the initiation of eruption. Therefore, we choose to use the extend of filament as a constraint to define an area within which the PIL is determined. The Sobel function (an embedded function of IDL) is then applied to the select area of the magnetogram and find PIL by looking for the pixels with the highest gradient and near zero field strength.

As an example, we first present the process of calculating the decay index of the eruptive filament/CME event on 2002 September 29. This filament erupted around 22:20 UT and the corresponding CMEs was observed by LASCO at 23:54 UT. Graphical representations of the details are illustrated in Figure 1. The top left shows an $\mathrm{H} \alpha$ image of the filament prior to its eruption at 20:31 UT obtained from the Global H $\alpha$ Network (GHN). The corresponding MDI magnetogram with the same FOV at 20:48 UT is shown in the top right panel. The PIL under the eruptive filament is plotted in white color on the top right panel. In the middle panel, we plot $\log \left(B_{t}\right)$ vs. $\log (h)$ for each pixel on the PIL. As mentioned before, the height range of 42 to $105 \mathrm{Mm}$ was used from extrapolated fields to calculate decay index. In logarithm space, these values are converted to 3.73 and 4.65 as marked by the two vertical lines in the middle panel. Within such a height range, the derived decay indexes are plotted in the bottom panel in an ascending order of their values but regardless their positions on the PIL. The lowest and highest values of the decay indexes are 1.31 and 1.75 , respectively. The mean decay index with one sigma uncertainty is $1.53 \pm 0.11$. Note that this is an event occurred in quiet sun. Not surprisingly we will see in the next section that this decay index is relatively small comparing with those of active regions.

\section{Statistical Study of Relationship Between Decay Indexes and CME Speed}

Motivated by the above analysis and previous studies (e.g., Liu 2008; Liu et al. 2010a), in this paper, we choose to compare the decay index with the eruption kinematics, in particular the linear speed of CMEs. As listed in Table 1, 38 events are selected for analysis. To minimize the projection effect on magnetic field extrapolation, only events not close to the solar limb (less than 60 degrees from the disk center in both E-W and N-S directions) were selected. Note that not all of these events are associated with filament eruptions. According 
to Jing et al. (2004), some CMEs were associated with flares but no filaments were involved in the eruptions. In some cases, the absence of filament eruption might be simply due to the data gap, or because that the material confined in filament channels is not dense enough to be observed as filaments (Martin 2000). In such cases, the magnetic configuration is not necessary to be different from that of events with filament eruptions. On the other hand, a number of eruptive filaments located in the quiet Sun do not incur detectable flares in X-ray emission. For those events without clearly observed filaments in $\mathrm{H} \alpha$ or EUV 304, the flare ribbons are used to outline the center of energy release area and the PIL is confined in such an area. In Table $\mathbb{1}$, decay indexes of 38 events are listed, as well as the linear CME velocities obtained from LASCO C2 catalog (http://cdaw.gsfc.nasa.gov/CME_list/index.html). For each event, the decay index is averaged in the height range of 42-105 Mm. As we can see in Figure 1, normally the decay index does not change too much within such a height range and the averaged value is pretty reasonable to present the magnetic gradient along the PIL.

The data points of these 38 events are presented in Figure 2 and fitted with a thirdorder polynomial function $\left(y=a+b x+c x^{2}+d x^{3}\right)$. Two distinct trends are found of the CME speed as a function of the decay index: 1) Below about $1000 \mathrm{~km} / \mathrm{s}$, the CME speed increases with decay index monotonically; 2) For CMEs with higher speed above $1000 \mathrm{~km} / \mathrm{s}$, the decay indexes are almost constant of 2.2. This upper limit is consistent with theoretical prediction of 2.0 (Kliem \& Török 2006) and the result of 2.25 from observational study (Liu et al. 2008). In five events, the erupted filaments were lied above quiet Sun regions and their decay indexes are among the smallest from 0.98 to 1.68. In Figure 2, these events are plotted with triangles. We are not able to draw any statistical conclusion using only five points, but these events appear to have relative small decay indexes indicating that the strapping fields of the quiet regions are relatively strong compared to the initiation force of the eruption.

\section{A Case Study of An Asymmetric Eruption}

Besides the fully eruptive events, partial and failed eruptions have attracted attention in recent years. The first event of failed eruption was studied by Ji et al. (2003). This event can be explained using the theory of kink instability and strong strapping field as simulated well by Török \& Kliem (2005).

A partial filament eruption on 2000 September 12, accompanied by the M1.0 flare and

\footnotetext{
${ }^{1}$ Some events were not associated with detectable flare emission in X-ray. The listed "flare time" is actually the time for $\mathrm{H} \alpha$ emission or filament eruption.
} 
Table 1: Overview of eruption events.

\begin{tabular}{|c|c|c|c|c|c|c|c|}
\hline Date & $\begin{array}{l}\text { Flare } \\
\text { Time }\end{array}$ & Location & Type & $\begin{array}{l}\text { CME } \\
\text { Time }\end{array}$ & $\begin{array}{l}\text { CME } \\
\text { Speed }\end{array}$ & $\begin{array}{l}\text { Decay } \\
\text { Index }\end{array}$ & $\begin{array}{r}\text { Flare } \\
\text { Magnitude }\end{array}$ \\
\hline 2000-Jul-14 & 10:10:00 & N18E00 & AR9077 & $10: 54: 07$ & 1674 & $1.98 \pm 0.19$ & X5.7 \\
\hline 2000-Sep-12 & 13:00:00 & S27W06 & AR9163 & $11: 54: 05$ & 1550 & $1.64 \pm 0.14$ & M1.0 \\
\hline 2000-Sep-16 & 05:05:00 & N14W13 & AR9165 & $05: 18: 14$ & 1215 & $2.17 \pm 0.18$ & M2.1 \\
\hline 2000-Nov-24 & 04:55:00 & N21W07 & AR9236 & $05: 30: 05$ & 1289 & $2.67 \pm 0.31$ & $\mathrm{X} 2.0$ \\
\hline 2000-Nov-25 & 00:59:00 & N09E32 & AR9240 & $01: 31: 58$ & 2519 & $2.62 \pm 0.16$ & M8.2 \\
\hline 2001-Apr-01 & 11:00:00 & N17W57 & AR9393 & $11: 26: 06$ & 1475 & $2.27 \pm 0.19$ & M2.1 \\
\hline 2001-Apr-10 & 05:06:00 & S22W20 & AR9415 & 05:30:00 & 2411 & $2.04 \pm 0.14$ & $\mathrm{X} 2.3$ \\
\hline 2001-Jun-15 & 10:00:00 & S26E41 & AR9502 & $10: 31: 33$ & 1090 & $2.36 \pm 0.20$ & M6.3 \\
\hline 2001-Sep-24 & 09:32:00 & S18E18 & AR9632 & $10: 30: 59$ & 2402 & $2.34 \pm 0.14$ & $\mathrm{X} 2.6$ \\
\hline 2001-Oct-09 & $10: 48: 00$ & S26E03 & AR9653 & 11:30:00 & 973 & $2.3 \pm 0.17$ & M1.4 \\
\hline 2001-Oct-19 & $16: 15: 00$ & N18W40 & AR9661 & $16: 50: 00$ & 901 & $1.93 \pm 0.09$ & $\mathrm{X} 1.6$ \\
\hline 2001-Oct-22 & $14: 27: 00$ & S19E13 & AR9672 & $15: 06: 05$ & 1366 & $2.2 \pm 0.08$ & M6.7 \\
\hline 2001-Nov-04 & 16:03:00 & N05W29 & AR9684 & $16: 35: 06$ & 1810 & $2.2 \pm 0.07$ & X1.0 \\
\hline 2002-Jan-28 & 10:00:00 & S23E15 & QS & $10: 54: 00$ & 524 & $1.5 \pm 0.08$ & C4.6 \\
\hline 2002-Apr-17 & 07:46:00 & S15W42 & AR9906 & $08: 26: 05$ & 1240 & $2.16 \pm 0.08$ & M2.6 \\
\hline 2002-Apr-22 & $22: 37: 00$ & S10W03 & QS & $00: 38: 00$ & 286 & $1.4 \pm 0.07$ & - \\
\hline 2002-May-21 & $21: 20: 00$ & N17E38 & AR9960 & $21: 50: 05$ & 853 & $1.85 \pm 0.12$ & M1.5 \\
\hline 2002-May-22 & 03:30:00 & S12W60 & QS & 03:50:05 & 1557 & $1.68 \pm 0.09$ & C5.0 \\
\hline 2002-Jul-07 & 17:00:00 & N08W49 & QS & 18:06:00 & 750 & $1.36 \pm 0.10$ & - \\
\hline 2002-Jul-29 & 07:13:00 & N32W40 & QS & 11:09:09 & 334 & $0.98 \pm 0.10$ & - \\
\hline 2002-Sep-29 & $22: 20: 00$ & S13E21 & QS & $23: 54: 00$ & 254 & $1.53 \pm 0.11$ & - \\
\hline 2002-Nov-09 & $13: 08: 00$ & S10W42 & AR10180 & $13: 31: 45$ & 1838 & $2.44 \pm 0.18$ & M4.6 \\
\hline 2003-May-29 & 00:51:00 & S07W46 & AR10365 & $01: 27: 12$ & 1237 & $2.32 \pm 0.19$ & $\mathrm{X} 1.2$ \\
\hline 2003-Jun-15 & $22: 35: 00$ & S16W39 & AR10380 & $23: 54: 05$ & 2053 & $2.39 \pm 0.11$ & $\mathrm{C} 2.1$ \\
\hline 2003-Oct-28 & 09:51:00 & S16E04 & AR10486 & $11: 30: 05$ & 2459 & $2.7 \pm 0.18$ & $\mathrm{X} 17$ \\
\hline 2003-Oct-29 & 20:40:00 & S17W10 & AR10486 & $20: 54: 05$ & 2029 & $2.55 \pm 0.08$ & $\mathrm{X} 10$ \\
\hline 2003-Nov-07 & $15: 42: 00$ & N09W08 & AR10696 & $15: 54: 05$ & 2237 & $2.34 \pm 0.09$ & $\mathrm{X} 2.0$ \\
\hline 2003-Nov-18 & 07:23:00 & N03E08 & AR10501 & 08:06:05 & 1223 & $2.2 \pm 0.21$ & M3.2 \\
\hline 2004-Jul-25 & 14:19:00 & N08W35 & AR10653 & $14: 54: 05$ & 1333 & $2.17 \pm 0.17$ & M1.1 \\
\hline 2004-Nov-10 & 01:59:00 & N09W49 & AR0696 & $02: 26: 05$ & 3387 & $2.2 \pm 0.14$ & $\mathrm{X} 2.5$ \\
\hline 2005-Jan-15 & $22: 16: 00$ & N13W04 & AR10720 & $23: 06: 50$ & 2861 & $2.34 \pm 0.10$ & $\mathrm{X} 1.6$ \\
\hline 2005-Jan-17 & 06:59:00 & N13W29 & AR10720 & 09:54:05 & 2547 & $2.29 \pm 0.14$ & $\mathrm{X} 3.8$ \\
\hline 2005-May-06 & 16:03:00 & S06E23 & AR10758 & $17: 28: 00$ & 1128 & $1.85 \pm 0.08$ & C8.5 \\
\hline 2005-Jul-09 & $21: 47: 00$ & N12W31 & AR10786 & $22: 30: 05$ & 1540 & $2.45 \pm 0.21$ & M2.8 \\
\hline 2005-Aug-28 & $10: 28: 00$ & N11E26 & AR10803 & $10: 56: 07$ & 1047 & $2.18 \pm 0.07$ & - \\
\hline 2005-Sep-13 & 19:19:00 & S11E17 & AR10808 & $20: 00: 05$ & 1866 & $1.82 \pm 0.07$ & $\mathrm{X} 1.5$ \\
\hline 2006-Dec-13 & 02:14:00 & S06W35 & AR10930 & 02:54:04 & 1774 & $2.06 \pm 0.19$ & X3.4 \\
\hline 2010-Aug-01 & 16:00:00 & W05N21 & QS & $23: 18: 00$ & 527 & $1.22 \pm 0.12$ & - \\
\hline
\end{tabular}


CME, is also of great interest. Wang et al. (2003) analyzed this event with multi-wavelength observations taken by the GHN, the Extreme ultraviolet Imaging Telescope (EIT) and LASCO. The authors tracked erupting filament to about 20 solar radii. In the present study, we revisit this event with a focus on the spatial distribution of decay index along the PIL. Using the line-of-sight magnetogram from MDI, we identified the PIL underlying the $\mathrm{H} \alpha$ filament before the eruption as shown in the left panel of Figure 3. The small shift between the filament and the PIL is due to the projection effect. The PIL is color-coded according to the values of decay index, and the mean decay index over the entire PIL is about 1.64. The white circle marks the non-erupting part of the filament. A visual inspection immediately reveals that the non-erupting part generally has smaller decay index than the erupting part. Panel (b) shows some samples of potential field lines superimposed on the line-of-sight MDI magnetogram and six given positions along the PIL. We then quantitatively examine the values of decay index at these six positions. The decay indexes of points 1 and 2 are 1.38 and 1.64, respectively, and the segment of the filament in between did not erupt Wang et al. 2003). As a comparison, the erupted portion of the filament lying above points 3 to 6 shows higher decay indexes, i.e., exceeding 1.7 and up to 1.9 when the altitude is higher than 42 $\mathrm{Mm}$. The result is consistent with the theoretical expectation that a slow decay of strapping field contributes more to confining the eruption while a quick decay of strapping field puts less constraint on the eruption.

\section{Discussion}

In this paper, we have analyzed 38 events that occurred not too close to the solar limb. The decay index of each event was calculated using the potential fields extrapolated from line-of-sight magnetograms. Combined with the linear velocities of CMEs obtained from the LASCO CME catalog, a good correlation is found. We noticed that the decay index increases monotonically with the CME speed up to $1000 \mathrm{~km} / \mathrm{s}$. For faster CMEs, the decay index tends to have no significant change. The upper limit of decay index is around 2.2 found by a polynomial fit to the data points. Our observational results are consistent with the modeling of Török \& Kliem (2007). In principle, the dynamics of an eruption may be affected by an initiation force $\left(F_{i}\right)$ to accelerate the erupting materials and the confined force $\left(F_{s}\right)$ due to the strapping field. Consequently, the kinetic energy of a CME is:

$$
\frac{1}{2} m v^{2}=\int\left(F_{i}-F_{s}\right) d h,
$$

i.e. 


$$
v=\sqrt{\frac{2 \int\left(F_{i}-F_{s}\right) d h}{m}},
$$

in which $m$ is mass of erupting materials, $v$ is the CME speed and $h$ is the height. When the strapping fields decay very fast, i.e., having large decay indexes, the $F_{s}$ is essentially zero. From the equations above, we can see that the CME speed is no longer correlated with decay index. This scenario is consistent with the flattened component of the curve plotted in Figure 2, in which the decay indexes are around 2.2 while the CME speed spreads in a wide range from $1000 \mathrm{~km} / \mathrm{s}$ to more than $3000 \mathrm{~km} / \mathrm{s}$. Let's consider another case that the strapping field dominates due to a very slow decay (small decay index) in which we have $\int\left(F_{s}\right) d h \geq \int\left(F_{i}\right) d h$, within a certain height range (for instance from $42 \mathrm{Mm}$ to $105 \mathrm{Mm}$ ). Consequently, no filament could erupt under the constraint of such a magnetic configuration. From previous numerical simulations, (e.g., Démoulin \& Aulanier 2010), the lower limit of decay index could be 1.1 for the failed eruption. Our fitted plot in Figure 2 indicates that at decay index equals to 1.0, CME speed approaches to zero. Above this threshold, the speed of CME is proportional to the square root of the net resistance force of $\Delta F=F_{i}-F_{s}$. According to the definition, the decay index $n=-d \log \left(B_{t}\right) / d \log (h)$. The negative sign in logarithm space indicates that $n$ is inversely proportion to the magnetic field strength and hence the restoring force, which in our case is $F_{s}$. We can define $F_{s}=\frac{A}{f(n)}$ and therefore Equation 2 becomes:

$$
v=\sqrt{\frac{2\left[\int F_{i} d h-\int\left(\frac{A}{f(n)}\right) d h\right]}{m}},
$$

in which $f(n)$ is some kind of function that relates $n$ and the force $F_{s}, A$ is a coefficient related to the magnetic tension. Equation 3 in principle resembles the curvature component (with CME speed less than $1000 \mathrm{~km} / \mathrm{s}$ ) of the plot in Figure2. We note that an exact fitting to this equation is not available due to the lack knowledge of the total mass of CME, the magnetic tension coefficient of $A$ and the exact form of $f(n)$. However, this equation reveals the relationship between decay index and CME dynamics with moderate speeds.

In addition, the case study of the asymmetric distribution of decay index of event on 2000 September 12 provides consist physical picture that the relative high decay index indicates weaker magnetic confinement in the strapping field. The filament component of failed eruption was associated with lower decay index representing a highly confined overlying arcade field. This result is consistent with that of Liu et al. (2010a).

Finally, we give limitations of our study. We note that only one magnetogram prior, 
usually two to three hours, to each eruption is used for extrapolation. Any rapid change of magnetic field, such as a flux emergence is not considered during this time period before eruptions. More detailed analysis with high cadence magnetograms, such as observations by SDO/HMI, will provide more accurate measurements of decay index. The results from potential field extrapolation need to be compared with those from other extrapolation methods, such as NLFFF. We used a height range of 42 to $105 \mathrm{Mm}$ as it was commonly used in previous studies (Liu 2008; Liu et al. 2010b). But different height selections could affect the calculation of decay index because the exact height ranges of strapping fields vary from event to event. The filament height, if can be measured accurately, may provide important hint to solve this problem. In addition, a sophisticated PIL selection tool can improve the accuracy of decay index measurements.

We would like to thank the referee for valuable comments. The CME catalog is generated and maintained by NASA and the Catholic University of America in cooperation with the Naval Research Laboratory. SOHO is a project of international collaboration between ESA and NASA. The Global High Resolution $\mathrm{H} \alpha$ Network is supported by NSF under grant ATM0839216. This work is supported by NSF-AGS-0832916, NSF-AGS-0849453 and NSF-AGS1153424. JJ is supported by NSF grant AGS 09-36665 and NASA grant NNX 11AQ55G.

\section{REFERENCES}

Antiochos, S. K., DeVore, C. R., \& Klimchuk, J. A. 1999, ApJ, 510, 485

Bateman, G. 1978, MHD instabilities (Cambridge, Mass., MIT Press, 1978, p.270)

Chen, P., Fang, C., \& Ding, M. 2001, Chinese Journal of Astronomy and Astrophysics, 1, 176

Chiu, Y. T., \& Hilton, H. H. 1977, ApJ, 212, 873

Démoulin, P., \& Aulanier, G. 2010, ApJ, 718, 1388

Fan, Y., \& Gibson, S. E. 2003, ApJ, 589, L105

—. 2007, ApJ, 668, 1232

Guo, Y., Ding, M. D., Schmieder, B., Li, H., Török, T., \& Wiegelmann, T. 2010, ApJ, 725, L38

Hood, A. W., \& Priest, E. R. 1981, Geophysical and Astrophysical Fluid Dynamics, 17, 297 
Ji, H., Wang, H., Schmahl, E. J., Moon, Y.-J., \& Jiang, Y. 2003, ApJ, 595, L135

Jing, J., Wiegelmann, T., Suematsu, Y., Kubo, M., \& Wang, H. 2008, ApJ, 676, L81

Jing, J., Yurchyshyn, V. B., Yang, G., Xu, Y., \& Wang, H. 2004, ApJ, 614, 1054

Kliem, B., \& Török, T. 2006, Physical Review Letters, 96, 255002

Liu, C., Lee, J., Jing, J., Liu, R., Deng, N., \& Wang, H. 2010a, ApJ, 721, L193

Liu, R., Gilbert, H. R., Alexander, D., \& Su, Y. 2008, ApJ, 680, 1508

Liu, S., Han, F., \& Fletcher, L. 2010b, ApJ, 709, 58

Liu, Y. 2008, ApJ, 679, L151

Martin, S. 2000, Solar Prominence Formation (Encyclopedia of Astronomy and Astrophysics, Edited by Paul Murdin, article 2281. Bristol: Institute of Physics Publishing, 2001)

Metcalf, T. R., De Rosa, M. L., Schrijver, C. J., Barnes, G., van Ballegooijen, A. A., Wiegelmann, T., Wheatland, M. S., Valori, G., \& McTtiernan, J. M. 2008, Sol. Phys., 247, 269

Moon, Y.-J., Choe, G. S., Wang, H., Park, Y. D., Gopalswamy, N., Yang, G., \& Yashiro, S. 2002, ApJ, 581, 694

Priest, E. R., \& Forbes, T. G. 2002, A\&A Rev., 10, 313

Schrijver, C. J., Elmore, C., Kliem, B., Török, T., \& Title, A. M. 2008, ApJ, 674, 586

Török, T., \& Kliem, B. 2005, ApJ, 630, L97

—. 2007, Astronomische Nachrichten, 328, 743

Török, T., Kliem, B., \& Titov, V. S. 2004, A\&A, 413, L27

Wang, H., Qiu, J., Jing, J., \& Zhang, H. 2003, ApJ, 593, 564 

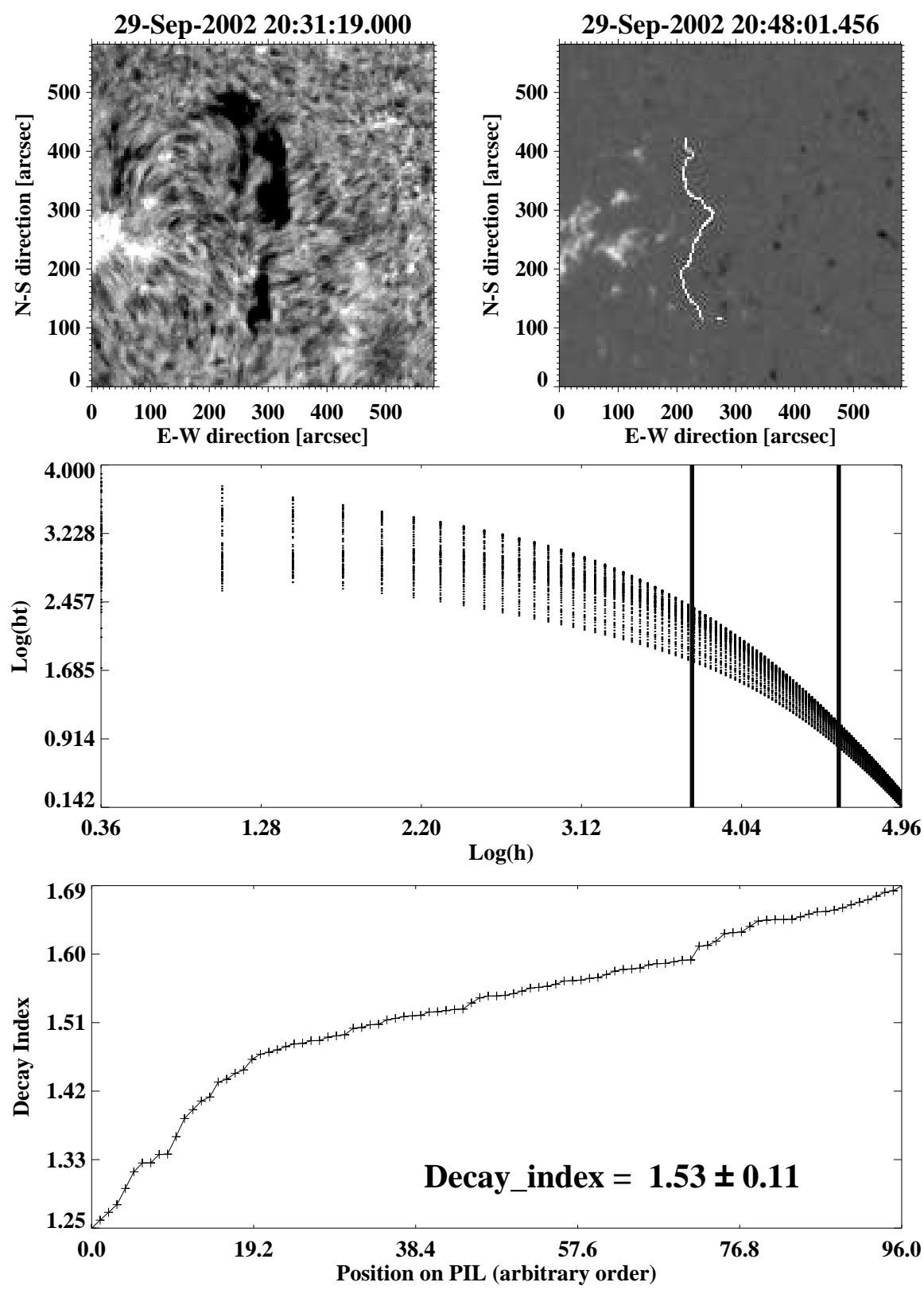

Fig. 1.- Top left panel: An $\mathrm{H} \alpha$ image showing the filament prior to its eruption observed on 2002 September 29. Top right panel: MDI magnetogram with PIL overploted (white curve). Middle panel: $\log \left(B_{t}\right)$ vs. $\log (h)$ along the PIL. The height range within which the decay index is calculated is marked by two vertical lines. Bottom panel: Derived decay indexes along the PIL in an ascending order. In average, the decay index for this event is 1.53. 


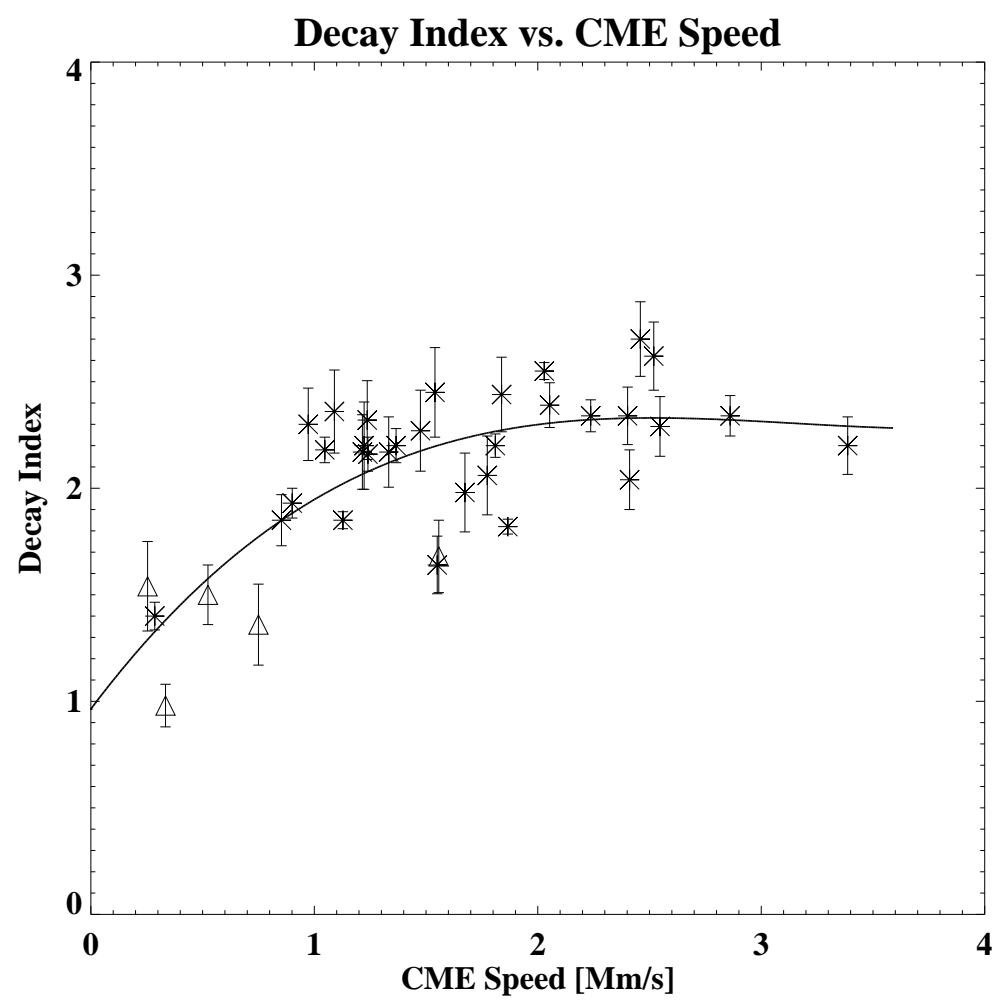

Fig. 2.- CME speed vs. the decay index for 38 CMEs associated with filament eruptions or two-ribbon flares events. All of the decay indexes are calculated within an height range of 42-105 Mm and averaged along the PIL, which are identified with the location of filaments or flare ribbons. The decay indexes for events occurred in the quiet Sun region are plotted with triangle symbol and all others are plotted with Asterisks. Using a 3-degree polynomial fit, an obvious trend is found which shows that the high speed CMEs correspond to higher decay index in strapping fields. 

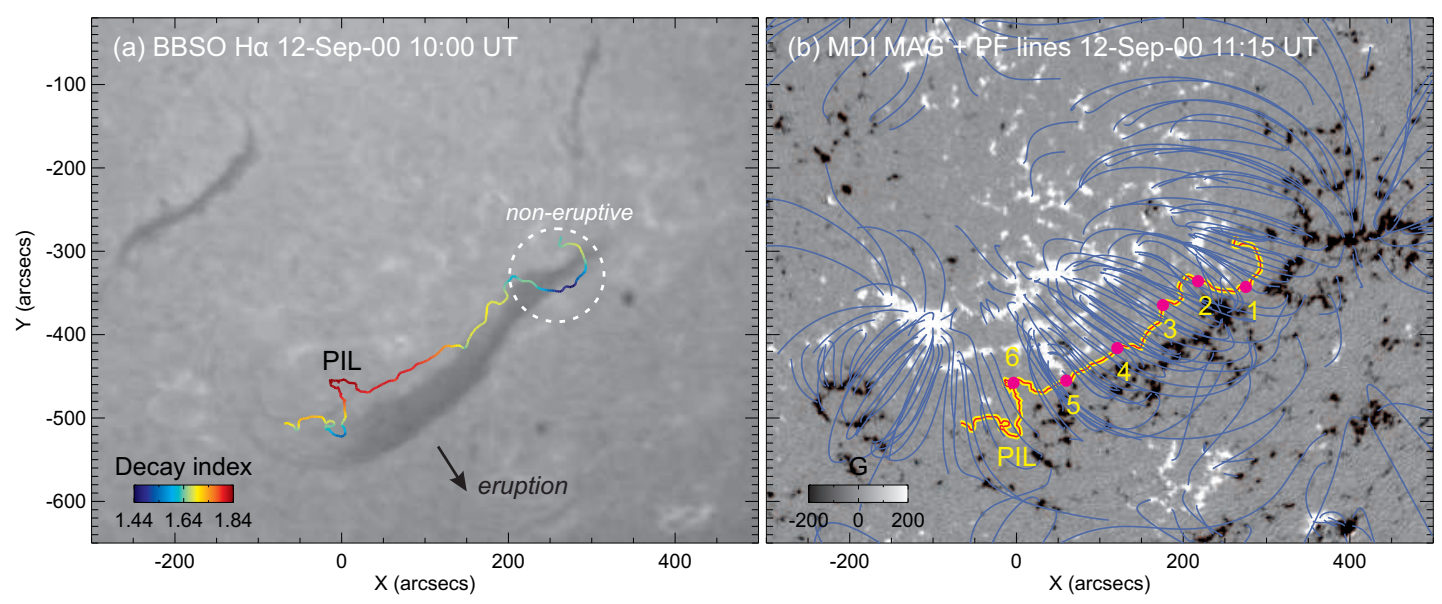

Fig. 3.- (a) Pre-eruption $\mathrm{H} \alpha$ image obtained from GHN, overplotted with the spatial distribution of the decay index along the PIL. The displacement between the filament and PIL is due to projection effect. This filament erupted partially. The white circle indicates the portion that did not erupt. (b) A corresponding line-of-sight MDI magnetogram taken half hour before the eruption, overplotted with the extrapolated potential field lines (blue). 